Nota Técnica/Technical Note

\title{
Coloraciones Ziehl-Neelsen y Safranina modificadas para el Diagnóstico de Cyclospora cayetanensis
}

\section{Modified Ziehl-Neelsen and modified Safranin staining for diagnosing Cyclospora cayetanensis}

\author{
Ana L. Galvan-Díaz ${ }^{1}$, Viviana Herrera-Jaramillo², Zila M. Santos-Rodriguez² y \\ Marcela Delgado-Naranjo ${ }^{2}$
}

1 Escuela de Microbiología, Universidad de Antioquia. Medellín, Colombia. agalvandiaz@yahooo.com

2 Investigadoras independientes. vivianahj@yahoo.com.ar, zmar6@yahoo.com.ar, andariel1819@yahoo.es

Recibido 8 Febrero 2007/Enviado para Modificación 29 Diciembre 2007/Aceptado Junio 32008

\section{RESUMEN}

Objetivo Comparar las coloraciones Ziehl-Neelsen modificada (ZNm) y Safranina modificada (Sm) en la detección de ooquistes de $C$. cayetanensis en muestras de materia fecal.

Metodología Se analizaron 100 muestras de materia fecal que fueron evaluadas previamente por coprológico directo y por concentración, posteriormente se realizaron las coloraciones ZNm y Sm para cada una de las muestras. Para la comparación de las dos técnicas se hicieron análisis estadísticos tomando como prueba oro el examen directo.

Resultados Se encontró una sensibilidad y especificidad del $95 \%$ y $98 \%$ para ZNm y del 90 \% y $100 \%$ para Sm respectivamente. El índice Kappa fue de 0,93, correspondiendo a un muy buen grado de concordancia entre las dos coloraciones.

Conclusiones Debido a la alta sensibilidad y especificidad encontradas en este estudio para Zm y Sm y al alto grado de concordancia entre ellas, cualquiera de las dos coloraciones puede ser utilizada para el diagnóstico de rutina de $C$. cayetanensis.

Palabras Claves: Técnica, diagnóstico, parásito (fuente: DeCS, BIREME).

\section{ABSTRACT}

Objective Comparing modified Ziehl-Neelsen (ZNm) and modified Safranin (Sm) staining for detecting $C$. cayetanensis oocysts in stool samples.

Methods The sample to be analysed consisted of 100 stool samples which had been previously evaluated by direct microscopic examination and concentration. Each sample was then ZNm and Sm stained. Microscope examination was used as gold standard for comparing both techniques by statistical analysis.

Results There was $95 \%$ sensitivity and 90\% specificity for ZNm and $98 \%$ sensitivity and $100 \%$ specificity for Sm. The Kappa index was 0,93 , signifying a very good degree of agreement between the two techniques. 
Conclusions The use of either of the two stains for diagnosing C. cayetanensis can be recommended due to the high sensitivity and specificity for $\mathrm{Zm}$ and $\mathrm{Sm}$ found in this study and to the high degree of agreement between them.

Key Words: Technique, diagnosis, staining, parasite, diarrhoea (source: MeSH, NLM).

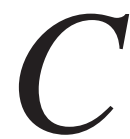

yclospora cayetanensis es un parásito intestinal emergente que en los últimos años ha sido un importante agente contaminante de aguas y alimentos, produciendo diarreas endémicas, epidémicas y la diarrea del viajero (1).La mayoría estos brotes son originados en países de Centro, Suramérica y el sur de Asia, lo que muestra la preferencia del parásito por las áreas tropicales y subtropicales aún cuando se ha visto que tiene una distribución cosmopolita (2). En América latina las prevalencias varían entre 2 y 20 \% y la mayoría de los casos se presentan después de épocas lluviosas y en los meses más calientes del año (3).

C. cayetanensis se caracteriza por presentar un ooquiste de forma esférica de 7,5 a 10 ìm de diámetro, estos son eliminados con la materia fecal de la persona infectada $(4,5)$. Este parásito se puede detectar observando los ooquistes en fresco mediante microscopía de luz, siendo este el método mas empleado en los laboratorios clínicos debido a que las estructuras son reconocidas sin necesidad de realizar tinciones especiales, sin embargo este método debe ser realizado por un observador experto ya que los ooquistes pueden ser confundidos fácilmente con otros parásitos como Blastocystis hominis, Endolimax nana y Cryptosporidium sp. (5-7); por tal motivo las coloraciones especiales, han sido adaptadas y utilizadas para el diagnóstico de C. cayetanensis, siendo ZiehlNeelsen modificada o Kinyoun la más común $(4,6)$. Debido a la gran variabilidad tintorial que presentan los ooquistes de $C$. cayetanensis varios autores han realizado modificaciones y reportado otras técnicas para teñir estas estructuras, como la coloración Safranina modificada la cual ha mostrado una alta capacidad y uniformidad tintorial de los ooquistes, convirtiéndose en una alternativa para el diagnóstico de $C$. cayetanensis (6-8).

\section{MATERIALES Y MÉTODOS}

Este trabajo es un estudio de tipo comparativo, para su desarrollo se emplearon 100 muestras de materia fecal preservadas en formalina al $10 \%$ provenientes de pacientes remitidos al Laboratorio de Parasitología de la Facultad de Medicina 
de la Universidad de Antioquia. De las 100 muestras, 20 eran positivas y 80 negativas para $C$. cayetanensis lo cual fue determinado previamente por examen directo y por la técnica de concentración con formol-éter. El procesamiento de las muestras y su observación se hizo a "ciegas” ya que estas fueron etiquetadas de tal manera que las realizadoras del estudio no tuvieran conocimiento de cuales muestras eran positivas y cuales negativas. Las placas coloreadas se observaron en toda su extensión con objetivo de 100X.

Métodos de coloración

Técnica de Zielh Neelsen Modificada. Se aplico el protocolo estandarizado por el Laboratorio de Parasitología, grupo GIEPI, de la Facultad de Medicina de la Universidad de Antioquia (5).

Técnica de Safranina Modificada. Se realizaron ensayos previos para la estandarización de la técnica reportada por Visvesvara utilizando muestras positivas para Cyclospora cayetanensis (8)

Metodología estadística. Los datos fueron organizados en hojas de cálculo y tablas mediante el programa Microsoft Excel 2003.

Se calculó la sensibilidad y especificidad de las coloraciones, tomando como prueba de oro el examen directo, se determinó el grado de concordancia de éstas a través del índice Kappa y los cocientes de probabilidad positivo y negativo.

\section{RESULTADOS}

Para el análisis de los resultados obtenidos con las dos coloraciones diagnósticas utilizadas, se establecieron dos categorías de muestras, positivas o negativas respecto a la presencia o ausencia de ooquistes de $C$. cayetanensis respectivamente, obteniendo resultados muy similares entre ambas coloraciones (Tabla 1).

Las dos coloraciones utilizadas se compararon con el directo (Tablas 2 y 3 ) encontrando una sensibilidad de $95 \%$ (19/20) y una especificad de 98,8 \% (79/ 80) para ZNm, y para la Sm una sensibilidad de $90 \%$ (18/20) y una especificidad de $100 \%(80 / 80)$. 
Se calcularon los Cocientes de Probabilidad Positivo ( $\mathrm{CP}+$ ) y Cociente de Probabilidad Negativo (CP-) de las dos coloraciones estudiadas, (los CPfueron expresados como 1 / CP-para facilitar la interpretación) encontrando que para la coloración ZNm un resultado positivo es 76 veces más probable en una persona con ciclosporiasis que en una persona sana, mientras que la probabilidad de encontrar un resultado negativo en una persona sana es 20 $(1 / 0,05)$ veces superior que en las personas que presentan la enfermedad. En el caso de la coloración de Sm la probabilidad de encontrar un resultado negativo en una persona sin ciclosporiosis es $10(1 / 0,1)$ veces superior que en los pacientes con la enfermedad. El CP+ de la safranina modificada no fue necesario de calcular debido a la especificidad del $100 \%$ de la prueba.

Tabla 1. Resultados de las técnicas, Directo, ZNm y Sm en la detección de ooquistes de $C$. cayetanensis en muestras de materia fecal

\begin{tabular}{|c|c|c|c|c|c|}
\hline Método & Muestras & Positivas & Negativas & $\begin{array}{l}\text { Falsos } \\
\text { Positivos }\end{array}$ & $\begin{array}{c}\text { Falsos } \\
\text { Negativos }\end{array}$ \\
\hline Directo & 100 & 20 & 80 & 0 & 0 \\
\hline $\begin{array}{c}\text { Ziehl Neelsen } \\
\text { modificado }\end{array}$ & 100 & 20 & 80 & 1 & 1 \\
\hline $\begin{array}{l}\text { Safranina } \\
\text { modificada }\end{array}$ & 100 & 18 & 82 & 0 & 2 \\
\hline
\end{tabular}

Tabla 2. Comparación de la coloración ZNm con el Examen Directo, en la detección de ooquistes de $C$. cayetanensis en muestras de materia fecal

\begin{tabular}{lcccc}
\hline & \multicolumn{4}{c}{ Examen Directo } \\
\cline { 2 - 5 } & & Positivo & Negativo & Total \\
Zlehl-Neelsen & Positivo & 19 & 1 & 20 \\
modificado & Negativo & 1 & 79 & 80 \\
\cline { 2 - 5 } & Total & 20 & 80 & 100 \\
\hline
\end{tabular}

ZNm: Ziehl Neelsen modificada

Tabla 3. Comparación de la coloración Sm con el Examen Directo, en la detección de ooquistes de C. cayetanensis en muestras de materia fecal

\begin{tabular}{ccccc}
\hline & \multicolumn{4}{c}{ Examen Directo } \\
\cline { 2 - 5 } & & Positivo & Negativo & Total \\
Safranina & Positivo & 18 & 0 & 18 \\
Modificada & Negativo & 2 & 80 & 82 \\
\cline { 2 - 5 } & Total & 20 & 80 & 100 \\
\hline
\end{tabular}

Sm: safranina modificada

Al determinar el grado de concordancia entre las dos coloraciones utilizadas se encontró un índice Kappa de 0,93, el cual equivale a un grado de acuerdo muy bueno entre las dos técnicas (según los márgenes propuestos por Landis y Koch). 


\section{DISCUSIÓN}

El examen directo en fresco de materia fecal ha sido el método más utilizado para realizar el diagnóstico de Cyclospora cayetanensis, sin embargo, en fechas recientes las coloraciones se han venido implementando para tal fin ya que facilitan la visualización de los ooquistes (2). Entre estas coloraciones la más empleada es ZNm con la cual los ooquistes de $C$. cayetanensis se tiñen de un color rojo oscuro o púrpura sobre un fondo azul. Sin embargo varios autores han reportado que no todos los ooquistes toman la coloración uniformemente $(9,10,15)$; en este estudio esta característica sumada al bajo número de ooquistes que son excretados en la materia fecal pudieron haber sido las causas del falso negativo encontrado (6), así mismo los artefactos presentes en las muestras pudieron haber influido en el falso positivo ya que también tomaron el colorante. A pesar de que esta coloración es la mas empleada se han realizado muy pocos estudios que evalúen la sensibilidad y especificidad de ZNm para detectar Cyclospora cayetanensis y ninguno que la compare con Sm. El Naggar reportó una sensibilidad del 100 \% con ZNm para la detección de C. cayetanensis, Isospora belli y Cryptosporidium parvum (4) y Rigo reportó una sensibilidad y especificidad del 100 \% de ZNm para Cryptosporidium parvum e Isospora belli (9).

La técnica de safranina modificada fue reportada por Visvesvara, su variación consistió en el calentamiento con horno microondas. En este estudio la mayoría de los ooquistes coloreados con esta técnica se observaron de color naranja brillante sobre un contraste azul, lo que permitió diferenciarlos de otras partículas con mayor facilidad, característica que pudo influir en que no se presentaran falsos positivos, coincidiendo con lo reportado por Visvesvara quien observó que el $98 \%$ de los ooquistes se colorearon uniformemente con la safranina, esto se atribuye al calentamiento con el microondas que aumenta la permeabilidad de los ooquistes al colorante (8). La especificidad y sensibilidad de esta coloración para C. cayetanensis no ha sido reportada por otros autores; en este estudio estos valores fueron muy altos y similares a los de ZNm, indicando que esta técnica tiene una alta validez a la hora de diagnosticar la presencia de ooquistes de $C$. cayetanensis.

La concordancia entre las dos coloraciones, hallada mediante el índice Kappa, fue muy buena indicando que ambas pruebas tienen la misma efectividad diagnóstica, lo anterior junto con los altos valores de sensibilidad y especificidad, hacen de estas dos técnicas unas buenas herramientas diagnósticas para la 
detección de Cyclospora cayetanensis en cualquier laboratorio clínico; la elección de alguna de las dos dependerá de los recursos y materiales disponibles en el laboratorio y la preferencia del personal •

Agradecimientos. Al Grupo interdisciplinario para el Estudio de las Parasitosis Intestinales-GIEPI de la Facultad de Medicina de la Universidad de Antioquia por el apoyo logístico para la realización de este estudio.

\section{REFERENCIAS}

1. Kimura K, Kumar S, Takemasa K, Ishibashi Y, Kawabata M, Belosevic M, et al. Comparison of three microscopic techniques for diagnosis of Ciclospora cayetenensis. FEMS Microbiology Letters. 2004; 238: 263-266.

2. Shields JM, Olson BO. Cyclospora cayetanensis: a review of an emerging parasitic coccidian. International Journal for Parasitology 2003; 33: 371-391.

3. Botero D. Parasitosis Humanas. 4a Edición. Medellín: Corporación para investigaciones Biológicas; 2003. p. 77-81.

4. El Naggar A. Studies on a newly emerging protozoal pathogen: Cyclospora cayetanensis. J. Egypt. Soc. Parasitol. 1999; 29: 575-586.

5. Agudelo S, Montoya M. Parásitos Intestinales Oportunistas Teoría y Práctica. 1a Edición. Medellín: Biogénesis fondo editorial; 2005. p. 31-35, 62.

6. Vázquez O, Jiménez R, Campos T, Valencia S, Romero R, Gamez V, et al. Infección por Cyclospora cayetanensis. Diagnóstico de Laboratorio. Revista Latinoamericana de Microbiología 2000; 42: 45-52.

7. Burstein S. Ciclosporosis: una Parasitosis Emergente (II). Diagnostico Microbiológico mediante una nueva técnica de coloración. Revista de Gastroenterología 2005; 25: 336-340.

8. Visvesvara GS, Moura H, Kovacs-Nace E, Wallace S, Eberhard L. Uniform Staining of Cyclospora Oocysts in fecal Smears by a Modified Safranin Technique with Microwave Heating. Journal of Clinical Microbiology 1997; 35(3): 730-733.

9. Rigo CR, Franco RM. Comparison between the modified Ziehl-Neelsen and Acid-Fast-Trichome methods for fecals screening of Cryptosporidium parvum and Isospora belli. Revista da Sociedad Brasileira de Medicina Tropical 2002; 35 (3): 209-214. 